\title{
Australian Bat Lyssavirus: examination of post-exposure treatment in NSW
}

\author{
Ben Ewald ${ }^{\mathrm{A}, \mathrm{C}}$ and David Durrheim ${ }^{\mathrm{B}}$ \\ ANewcastle University, New South Wales \\ ${ }^{\mathrm{B}}$ Hunter New England Population Health, \\ Hunter New England Area Health Service \\ ${ }^{\mathrm{C} C o r r e s p o n d i n g ~ a u t h o r . E m a i l: ~ b e n . e w a l d @ n e w c a s t l e . e d u . a u ~}$
}

\begin{abstract}
Ten years after the recognition of Australian Bat Lyssavirus, it is timely to review the occurrence of the virus in native microbat and flying fox species in Australia, and the effectiveness of post-exposure treatment in humans. Differences between post-exposure treatment protocols adopted by state and territory health departments were examined. In Queensland and the United States of America, post-exposure treatment is withheld in people who are bitten by bats that subsequently test negative for ABLV and rabies, respectively. The good outcomes from these protocols support the revised NSW policy, which delays post-exposure treatment for up to 48 hours for minor exposures while awaiting bat test results. Post-exposure treatment can be withheld or ceased if the bat test result is negative.
\end{abstract}

Two deaths have been caused by Australian Bat Lyssavirus (ABLV) infection in Australia. The clinical disease was a progressive encephalitis indistinguishable from rabies. ABLV is phylogenetically closely related to rabies virus, lyssavirus 1 . Since ABLV was recognised, all state and territory health departments in Australia have adopted protocols to administer post-exposure treatment (PET) to people bitten or scratched by bats, but there are jurisdictional differences. As it is a decade since the first human case was diagnosed, it is timely to review the NSW protocol against current practice in other jurisdictions, contemporary epidemiological understanding of ABLV, and the evidence for effectiveness of post-exposure treatment.

\section{ABLV in Australia}

Australian Bat Lyssavirus, genotype 7 of the lyssavirus genus, is a member of the family Rhabdoviridae that shares many serotypic, antigenic and molecular sequence features with classical rabies virus. ${ }^{1}$ It was first reported in July 1996 in a black flying fox (Pteropus alecto) from Ballina, NSW, and has subsequently been confirmed in five species of Australian bat, four species of flying fox (suborder Megachiroptera, genus Pteropus) and one species of insectivorous bat (suborder Microchiroptera, Saccolaimus flaviventris). ${ }^{2}$ Two Queensland women are known to have succumbed to disease caused by ABLV: one woman from Rockhampton died in November 1996, within 5 weeks of a scratch from a microbat, probably a yellow-bellied sheath-tailed bat ( $S$. flaviventris); and the second woman, from Mackay, died in December 1998, more than two years after a bite from a flying fox. Their ante-mortem clinical presentation was indistinguishable from classic rabies infection. ${ }^{3,4}$

Australia has five flying foxes in the genus Pteropus. They eat fruit and live in large colonies, often with multiple species roosting together. Australia also has a diversity of small insectivorous microbats, with approximately 80 species recorded. In NSW, the grey headed flying fox ( $P$. poliocephalus) inhabits predominantly coastal and mountainous regions; the black flying fox (P. alecto) is found mainly in the northern coastal area; and the little red flying fox (P. scapulatus) has a range that extends further west, encompassing most of the state. ${ }^{5}$ The microbat, $S$. flaviventris, is distributed throughout Australia, except South Australia and the southern parts of Western Australia.

\section{Prevalence in Queensland and NSW}

To allow for an assessment of the risk, it is necessary to establish the prevalence of ABLV among flying foxes and microbats. Prevalence data from bats submitted through an arrangement of Queensland public health units with Queensland Scientific Services during July 1998 to February 2006, and from bats submitted through an arrangement of NSW public health units with Australian Animal Health Laboratories between 1995 and 2005 are presented in Table 1.

It is important to note that the four microbats from NSW that tested positive for ABLV were all detected in the first quarter of 1999, but were not identified to the species level. These are similar findings to a large screening program conducted in partnership with Department of Primary Industries in Queensland from June 1996 to March $2002 .{ }^{6}$ Among submitted ill or injured animals, 69/974 (7.1\%) of flying foxes and $5 / 158(3.2 \%)$ of microbats were ABLV positive using direct fluorescent antibody testing (DFAT). 
All five positive microbats were $S$. flaviventris, and these were five of the seven $S$. flaviventris specimens tested. In another large survey of caught wild bats, none of 475 wildcaught flying foxes were DFAT positive. ${ }^{7}$ Eight of $266(3 \%)$ wild-caught flying foxes were antibody positive. Of 318 wild-caught microbats, none were DFAT positive while 9 $(2.8 \%)$ were antibody positive.

The only microbat found to be DFAT positive, the yellow bellied sheath tail (S. flaviventris), is secretive and rarely seen by humans, and appears to have a high prevalence of infection. It has its own sub-strain of ABLV virus, which was responsible for a death in a bat handler. Four other species of small bats have been found to have antibodies to ABLV. There is uncertainty about whether these species can transmit infection. The antibodies may represent seroconversion without infectivity or response to an insect rhabdovirus.

\section{Exposures in Queensland}

In a series of bat exposures reported in Brisbane, those exposed to bat bites were largely members of the public who approached a bat (55\%) and professional or volunteer bat handlers $(20 \%){ }^{8}$ In $15 \%$ of cases, the bat initiated contact. Over the last 10 years, the Brisbane Southside Public Health Unit has administered over 300 courses of PET. Comparable figures for NSW are not collated.

\section{Laboratory testing of bats}

Queensland Health uses the Queensland Scientific Services laboratory in Brisbane for testing the brains of submitted bats for ABLV. All other states use the Australian Animal Health Laboratory in Geelong. Both laboratories use a DFAT on brain impression smears and also perform confirmatory polymerase chain reaction tests on all specimens. Complete concordance has been documented between these tests. ${ }^{9}$ International experts state that either test alone is considered adequate for confirmation of rabies infection. ${ }^{10,11}$ The Australian Animal Health Laboratory also performs a DFAT on salivary gland tissue. Extensive experience with lyssavirus 1 has indicated that PET is unnecessary if the test results in the bat are negative (C Rupprecht, pers comm).

\section{Effectiveness of post-exposure treatment}

Of the 53 positive bats submitted by public health units (Table 1), it is not known how many bit more than one person. However, it would be safe to estimate that the number of people exposed to known positive bats is between 50 and 100. Experience with rabies in countries where vaccine is not available shows that not everyone bitten by a rabid dog develops disease, so infection is unlikely to be universal in humans following a bite by a positive bat. ${ }^{12}$

Cross protection offered by rabies vaccine against ABLV is supported by two published papers. Tests conducted at the Centers for Disease Control and Prevention, Atlanta, Georgia, in mice vaccinated with four different rabies vaccines, found that all five mice challenged with ABLV survived. These tests did not include the vaccine currently used in Australia; however, they provided the basis for the current PET recommendation in Australia. ${ }^{1}$ A second study published in 2005, examined cross protection against both ABLV and European Bat Lyssavirus. It demonstrated protection in only 15 of 19 mice challenged with ABLV. ${ }^{13}$ Cross neutralisation with antibodies from 52 volunteers immunised with the human diploid cell vaccine ( $\mathrm{HDCV}$, as used in Australia) against various lyssaviruses, including ABLV, showed similar levels of neutralisation against all viruses, especially in low and mid level responders. As the vaccine was not fully protective in mice, and there are considerable interspecies differences in the virulence of the various lyssavrius genotypes, this is not convincing evidence of the vaccine's efficacy against ABLV in humans. Nevertheless, there has not been a failure of properly administered PET for rabies in the United States of America since 1979. ${ }^{10}$

It is reassuring that there is no evidence of development of encephalitis documented among individuals administered PET. However, this does not guarantee $100 \%$ efficacy in preventing ABLV given the occasional prolonged incubation period of lyssavirus infection and the current incomplete documentation of administration and follow up of individuals initiated on PET.

In locations where treatment was withheld due to bats

Table 1. Australian Bat Lyssavirus prevalence in Queensland bats submitted to Queensland Scientific Services, July 1998 to February 2006, and NSW bats submitted to Australian Animal Health Laboratories, January 1995 to December 2005

\begin{tabular}{|c|c|c|c|c|c|c|c|c|c|}
\hline & \multicolumn{3}{|c|}{ Queensland } & \multicolumn{3}{|c|}{ NSW } & \multicolumn{3}{|c|}{ Total } \\
\hline & \multirow{2}{*}{$\begin{array}{c}\text { Tested } \\
n\end{array}$} & \multicolumn{2}{|c|}{ Positive } & \multirow{2}{*}{$\begin{array}{c}\text { Tested } \\
n\end{array}$} & \multicolumn{2}{|c|}{ Positive } & \multirow{2}{*}{$\begin{array}{c}\text { Tested } \\
n\end{array}$} & \multicolumn{2}{|c|}{ Positive } \\
\hline & & $n$ & $\%$ & & $n$ & $\%$ & & $n$ & $\%$ \\
\hline Flying foxes & 485 & 30 & 6.2 & 249 & 18 & 7.2 & 734 & 48 & 6.5 \\
\hline Microbats & 115 & 1 & 0.9 & 68 & 4 & 5.9 & 183 & 5 & 2.7 \\
\hline Total & 600 & 31 & 5.2 & 317 & 22 & 6.9 & 917 & 53 & 5.8 \\
\hline
\end{tabular}


testing negative, there have so far been no negative outcomes. Brisbane Southside Public Health Unit reported that of 246 exposures managed from 1996 to 2003, PET was either withheld or ceased for 65 individuals after a negative bat result. ${ }^{8}$ Updating this data in 2005 provided an additional 59 people exposed to bat bites or scratches, of whom 26 had the bat tested (all were negative) and 24 were not administered PET (Jarvinin, pers comm.).

\section{Australian post-exposure treatment protocols}

In Australia, a variety of protocols are available to guide PET following a bat exposure. These include the National Guidelines, the 8th edition of the Immunisation Handbook and various state and territory notifiable disease guidelines. ${ }^{14,15}$ Only South Australia and Tasmania do not have their own guidelines and defer exclusively to the National Guidelines for PET. Protocols share many features, for example: similar approaches to all bat exposures whether flying foxes or microbats; emphasis on the importance of immediate rigorous wound cleaning; using the same doses and schedules for immunoglobulin (HRIG) and rabies HDCV; and omitting HRIG where more than 7 days has elapsed since the first vaccine dose.

All protocols emphasise the necessity of testing the bat for ABLV. However, protocols differ notably in two aspects: the influence of bat brain testing for ABLV on PET and the influence of exposure type on PET. The Queensland, Australian Capital Territory and Northern Territory protocols explicitly state that PET should be withheld or ceased if the implicated bat's brain tests negative for ABLV. The Victorian protocol and NHMRC Immunisation Handbook also indirectly advocate this approach. The National Guideline defers this decision to local public health units. However, the former NSW protocol explicitly and uniquely stated that PET is not affected by the results of tests on the bat.

Protocols in NSW, Australian Capital Territory and Victoria distinguish between severe and mild exposures. Severe bites are: bites on the face or neck; bites by a sick or abnormally behaving bat; or unprovoked or multiple bites. These states advocate immediate initiation of PET for a severe bite, but a delay of up to $48 \mathrm{~h}$ for other exposures. Another inconsistency is HRIG administration. Queensland and Victoria recommend excluding HRIG if the bite occurred more than 12 months previously, while the NHMRC Immunisation Handbook suggests administration in this circumstance. Other protocols are silent on this issue.

\section{Discussion}

ABLV has never been found in a bat caught during wildlife surveys, but has been present in the brains of approximately $6 \%$ of bats with human contact. Although wildlife surveys suggest that the risk from small bats is less than from large bats, there is sufficient uncertainty that the recommendation to regard all bats as infective should remain.

Laboratory tests on the brain of the bat, when available, can reliably detect the presence of Lyssavirus as supported by the concordance of different tests. Evidence from Queensland and the USA suggests that where a bat brain tests negative, it is not necessary to administer PET. There was no disease in the 89 people not given PET following the Queensland protocol, and similar positive experience with the same testing and management protocol following animal bites for thousands of people each year in the USA. ${ }^{10}$

Evidence to support rabies protocols distinguishing severe and mild bites comes from reports of PET failures after dog bites in Thailand, in which a common feature was bites to the head or neck. ${ }^{16,17}$ PET failure is more likely after multiple dog bites, and the incubation time is shorter for bites closer to the brain, supporting the suggestion for PET to be initiated more promptly after such bites. There is a relative paucity of evidence from animal studies on the effectiveness of rabies vaccine against ABLV as the research was conducted only in small numbers of mice; however, further work in this area is currently underway.

Unfortunately there is currently no aggregation of data on the number or completion of PET courses in NSW, although vaccine supply data suggests up to 130 per year.

The current NSW guideline requests testing of all bats. Although this could lead to under-reporting of bites by carers who do not want the animal destroyed, the use of bat testing to guide PET decisions will result in some savings through reduced PET administration.

\section{Conclusion}

The lack of adverse outcomes in Queensland and the USA supports withholding PET following bat exposures in nonsevere bites if the bat tests negative. There is no direct evidence of the acceptable length of delay while waiting for a laboratory result, however 48 hours seems reasonable. Bats should be submitted without delay for ABLV testing directly to AAHL or QSS. For any bite PET may be suspended on the basis of a negative laboratory bat test.

It would be sensible to collate bat exposure and PET experience across NSW and Australia, including matching the bat results to the public health unit patient record. Public education on avoiding bat contact, and what to do if it occurs, remains the mainstay of preventing human ABLV infection. For a map of the distribution of big bats in Australia, see http://www.newscientist.com/article/mg 16021635.200-bats-out-of-hell.htm. ${ }^{18}$ 


\section{Acknowledgements}

We are grateful to NSW Department of Primary Industry and Queensland Health scientific services staff who provided the data for Table 1, staff from AAHL who provided the details on the diagnostic methods, and from PHUs in all Australian states and territories who provided copies of their public health protocols.

\section{References}

1. Hooper P, Lunt R, Gould A, Samaratunga H, Hyatt A, Gleeson L et al. A new Lyssavirus: the first endemic rabies related virus recognised in Australia. Bull Inst Pasteur 1997; 95: 209-18. doi:10.1016/S0020-2452(97)83529-5

2. Gould AR, Hyatt A, Lunt R, Kattenhelt J, Hengstberger S. Characterisation of a novel lyssavirus isolated from Pteropid bats in Australia. Virus Res 1998; 54: 165-87. doi:10.1016/S0168-1702(98)00025-2

3. Fraser G, Hooper P, Lunt R, Gould A, Gleeson L, Hyatt A. Encephalitis caused by a lyssavirus in fruit bats in Australia. Emerg Infect Dis 1996; 2: 327-31.

4. Hanna J, Carney I, Smith G, Tannenberg A, Deverill J, Botha J et al. Australian bat lyssavirus infection: a second human case, with long incubation period. Med J Aust 2000; 172: 597-9.

5. Hall L, Richards G. Flying foxes fruit and blossom bats of Australia. Sydney: UNSW Press, 2000.

6. Barrett J. Australian Bat Lyssaviruses. Thesis, University of Queensland, 2005.

7. Field H. The ecology of Hendra virus and Australian Bat Lyssavirus. Thesis, University of Queensland, 2005.

8. Young M, McCall B. Trends in potential exposure to Australian bat Lyssavirus in south east Queensland, 1996 to 2003. Commun Dis Intell 2004; 28: 258-60.
9. Warrilow D, Harrower B, Smith I, Field H, Taylor R, Walker C et al. Public health surveillance for ABLV in Queensland, Australia 2000, 2001. Emerg Infect Dis 2003; 9: 262-4.

10. Rupprecht C, Gibbons R. Prophylaxis against rabies. N Engl $J$ Med 2004; 351: 2626-35. doi:10.1056/NEJMcp042140

11. Jackson A, Warrell M, Rupprecht C. Management of rabies in humans. Clin Infect Dis 2003; 36: 60-3. doi:10.1086/344905

12. Cleaveland S, Fevre EM, Kaare M, Coleman PG. Estimating human rabies mortality in the United Republic of Tanzania from dog bite injuries. Bull World Health Organ 2002; 80: 304-10.

13. Brookes S, Parsons G, Johnson N, McElhinney L, Fooks A. Rabies human diploid cell vaccine elicits cross-neutralising and cross-protecting immune response against European and Australian bat lyssaviruses. Vaccine 2005; 23: 4101-9. doi:10.1016/j.vaccine.2005.03.037

14. Commonwealth Department of Health and Ageing. Australian Bat Lyssavirus Information for medical practitioners. Canberra: Commonwealth Department of Health and Ageing, 2001.

15. National Health and Medical Research Council. Australian Immunisation Handbook, 8th edn. Canberra: National Health and Medical Research Council, 2003.

16. Wilde H, Choomkasien P. Failure of rabies postexposure treatment in Thailand. Vaccine 1989; 7: 49-52. doi:10.1016/0264-410X(89)90010-8

17. Wilde H, Sirikawin S. Failure of postexposure treatment of rabies in children. Clin Infect Dis 1996; 22: 228-32.

18. Anderson I. Bats out of hell. New Scientist 1998; 2163: 40. Available from http://www.newscientist.com/article/mg 16021635.200-bats-out-of-hell.htm 